\title{
ACEITAÇÃO DE FITOTERÁPICOS POR PRESCRITORES DA ATENÇÃO PRIMÁRIA À SAÚDE
}

ACCEPTANCE OF PHYTOMEDICINES BY PRIMARY HEALTH CARE PRESCRIBERS

ACEPTACIÓN DE FITOTERÁPICOS POR PRESCRIPTORES DE LA ATENCIÓN PRIMARIA DE SALUD

Ariel Átila Pontes Soares ${ }^{1}$

Ana Cecília Rodrigues e Silva ${ }^{2}$

Joaquim Horácio de Araújo Neto ${ }^{3}$

André Luiz Cunha Cavalcante ${ }^{4}$

Olindina Ferreira Melo ${ }^{5}$

Rafaelly Maria Pinheiro Siqueira 6

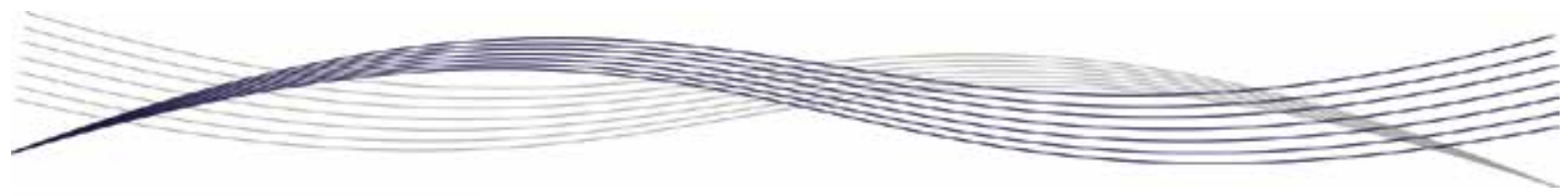

RESUMO

Palavras-chave:

Fitoterapia; Prescritores;

Atenção Primária à Saúde;

Centros de Saúde.

Keywords:

Phytotherapy; Prescribers;

Primary Health Care; Health

Centers.

Palabras clave:

Fitoterapia; Prescriptores;

Atención Primaria de Salud;

Centros de Salud.

Submetido:

28/05/2018

Aprovado:

$12 / 11 / 2018$

Autor(a) para Correspondência:

Rafaelly Maria Pinheiro Siqueira R. Zuca Accioly, 633, apto. 202 Manoel Dias Branco

Fortaleza (CE)

CEP: 60191-335

E-mail:

rafaellysiqueira@gmail.com
Esta pesquisa analisou a aceitação e o uso prescritivo de fitoterápicos em Unidades Básicas de Saúde (UBS) de Viçosa do Ceará-CE. Trata-se de estudo exploratório, descritivo, com abordagem quantiqualitativa. Os dados foram coletados por meio de entrevista semiestruturada com 12 profissionais que atuam na Atenção Primária à Saúde (APS). Os resultados são apresentados em tabela, figura e categorização de falas. Os participantes do estudo foram 6 médicos e 6 enfermeiros; houve prevalência do sexo feminino $e$ a idade variou de 30 a 39 anos. Identificou-se que todos os profissionais conhecem e prescrevem os fitoterápicos (50\% com muita frequência $e$ $50 \%$ com frequência regular) e 11 dos 12 participantes (91,7\%) relataram a indisponibilidade dos fitoterápicos na unidade de farmácia viva como o principal fator limitante para sua prescrição. Apenas 1 profissional $(8,3 \%)$ relatou ter conhecimento insuficiente sobre os fitoterápicos. As formulações mais prescritas foram xaropes, cápsulas de maracujá e elixir de aroeira. Constatou-se que os fitoterápicos são relevantes na APS, mas demandam maior investimento e qualificação profissional para ampliar os benefícios proporcionados à população.

\footnotetext{
1. Farmacêutico graduado pelo Centro Universitário Inta (Uninta). Sobral (CE), Brasil.

2. Enfermeira. Especialista em Saúde da Família pela Universidade do Vale do Acaraú (UVA). Sobral (CE), Brasil.

3. Aluno de Graduação em Farmácia no Uninta. Sobral (CE), Brasil.

4. Médico. Mestre em Ciências Médicas pela Universidade Federal do Ceará (UFC). Fortaleza (CE), Brasil.

5. Farmacêutica. Mestre em Bioquímica. Professora do Uninta. Sobral (CE), Brasil.

6. Farmacêutica. Mestre em Farmacologia. Professora do Uninta. Sobral (CE), Brasil.
} 


\section{ABSTRACT}

This research analyzed the acceptance and prescriptive use of phytomedicines in Brazilian primary health care centers (UBS) in Viçosa do Ceará, Ceará, Brazil. This is an exploratory, descriptive, study with a quantiqualitative approach. Data were collected by using a semi-structured interview with 12 professionals who work in primary health care $(P H C)$. The results are presented in table, figure, and speech categorizations. The study participants were 6 physicians and 6 nurses; there was a prevalence of women and age ranged from 30 to 39 years. It was identified that all professionals know and prescribe the phytomedicines (50\% very frequently and 50\% regularly) and 11 out of the 12 participants (91.7\%) reported the unavailability of phytomedicines in the live pharmacy unit as the main limiting factor for their prescription. Only 1 professional (8.3\%) reported having insufficient knowledge about phytomedicines. The most prescribed formulations were syrups, passion fruit capsules, and aroeira elixir. It was found that phytomedicines are relevant in PHC, but they require greater investment and professional qualification to increase the benefits provided to the population.

\section{RESUMEN}

Esta investigación analizó la aceptación y el uso prescriptivo de fitoterápicos en Unidad Básicas de Salud (UBS) en Viçosa do Ceará, Ceará, Brasil. Este es un estudio exploratorio, descriptivo, con un abordaje cuanticualitativo. Los datos se recopilaron mediante entrevista semi-estructurada con 12 profesionales que trabajan en la Atención Primaria de Salud (APS). Los resultados se presentan en tabla, figura y categorización de hablas. Los participantes del estudio fueron 6 médicos y 6 enfermeros; hubo prevalencia de mujeres y la edad varió de 30 a 39 años. Se identificó que todos los profesionales conocen y prescriben los fitoterápicos (50\% con mucha frecuencia y $50 \%$ con frecuencia regular) y 11 de los 12 participantes (91,7\%) informaron la indisponibilidad de fitoterápicos en la unidad de farmacia viva como principal factor limitante para su prescripción. Solo 1 profesional (8,3\%) informó tener un conocimiento insuficiente acerca de los fitoterápicos. Las formulaciones más prescritas fueron jarabes, cápsulas de maracuyá y elixir de aroeira. Se constató que los fitoterápicos son relevantes en la APS, pero requieren mayor inversión y calificación profesional para aumentar los beneficios proporcionados a la población.

\section{INTRODUÇÃO}

0 conhecimento sobre o uso de plantas com fins terapêuticos faz parte de diferentes culturas do mundo ao longo da história humana. Tendo em vista sua flora diversificada e sua multiplicidade étnica e cultural, o Brasil é detentor de tradição no uso de plantas medicinais, motivo que leva a fitoterapia a ser respeitada como recurso terapêutico integrativo e complementar à saúde, contando com o apoio de políticas públicas e normatizações específicas que buscam institucionalizar essa prática no Sistema Único de Saúde (SUS) ${ }^{1}$.

Com o reconhecimento do uso de fitoterápicos com finalidade profilática e curativa pela Organização Mundial da Saúde (OMS), o Brasil iniciou um processo de validação dessa prática ${ }^{2}$. A Política Nacional de Práticas Integrativas e Complementares (PNPIC) e a Política Nacional de Plantas Medicinais e Fitoterápicos (PNPMF) foram implantadas em 2006; elas contemplam diretrizes, ações e responsabilidades nas 3 esferas de governo para a oferta de serviços e produtos, culminando na criação do Programa Nacional de Plantas Medicinais e Fitoterápicos, em 2009. A abertura de novas opções terapêuticas no âmbito do SUS simbolizou o resgate e a revalorização do conhecimento popular, pautado por saberes científicos que garantem sua eficácia e segurança $a^{3-4}$.

Entre os programas direcionados à saúde pública envolvendo o uso de plantas medicinais, o Projeto Farmácias Vivas, idealizado em 1983 pelo farmacêutico Francisco José de Abreu Matos ${ }^{5}$, da Universidade Federal do Ceará (UFC), tornou-se referência no Nordeste e, posteriormente, em todo o Brasil. Hoje, - Programa Farmácias Vivas é direcionado à saúde pública e suas plantas possibilitam o tratamento de aproximadamente $80 \%$ das enfermidades mais comuns nas populações de baixa renda. Sua essência reside no uso de plantas medicinais como matéria-prima na fabricação de medicamentos com teor farmacológico considerável e boa relação custo/benefício ${ }^{3,5}$.

Contudo, embora se evidencie sua importância diante do cenário nacional da saúde pública e da emergência de novas alternativas de tratamento e controle de enfermidades, essa prática ainda enfrenta dificuldades e/ou desafios em sua implantação no SUS $^{3,5-8}$. 
Com base no Programa Farmácias Vivas, o Laboratório Fitoterápico de Viçosa do CearáCE foi inaugurado em 2002, segundo o conceito de Farmácia III. Nele são manipulados diversos fitoterápicos, oriundos da flora medicinal da região, para tornar viável a conversão do uso tradicional em uso convencional (em termos técnico-científicos) de plantas classificadas como medicinais. A iniciativa teve por objetivo proporcionar ao município: redução de gastos com medicamentos industrializados; minimização de danos colaterais causados pelo uso de medicamentos industrializados; respeito ao conhecimento popular; integração e equilíbrio entre o meio ambiente e a população que adota essa prática terapêutica, entre outros benefícios ${ }^{9}$.

Nesse contexto, diante da facilidade de acesso e obtenção desses medicamentos naturais no Laboratório Fitoterápico de Viçosa do Ceará, esta pesquisa analisou a aceitação e o uso prescritivo de fitoterápicos por parte de prescritores em unidades básicas de saúde (UBS) do município.

Este estudo se justifica por verificar o nível de aceitação de fitoterápicos entre profissionais da atenção primária à saúde (APS) responsáveis pela prescrição, buscando identificar os fatores que influenciam tal opção. A iniciativa se mostra relevante por proporcionar dados sobre o uso de fitoterápicos e o quadro procedimental e metodológico dessa prática no âmbito da saúde pública.

\section{METODOLOGIA}

Este é um estudo exploratório, descritivo, com abordagem quantiqualitativa. Foi realizado em 6 UBS, abrangendo as zonas urbana e rural de Viçosa do Ceará, entre fevereiro e junho de 2015.

Mostraram-se elegiveis para inclusão na pesquisa os médicos e enfermeiros responsáveis pela prescrição de fitoterápicos nessas UBS. Sua participação foi confirmada mediante assinatura de termo de consentimento livre e esclarecido. Assim, a amostra totalizou 12 profissionais $(6$ médicos e 6 enfermeiros). 0 estudo foi aprovado pelo Comitê de Ética em Pesquisa da UFC, sob o Parecer $n$. $041205 / 2015$.

0 s dados foram coletados por meio de entrevista semiestruturada (gravada e transcrita na íntegra), norteada por roteiro dividido em 2 blocos: informações gerais dos entrevistados; e questões relativas à prescrição de fitoterápicos.

Os achados quantitativos são apresentados

\author{
...o uso de \\ fitoterápicos \\ e o quadro \\ procedimental e \\ metodológico dessa \\ prática no âmbito \\ da saúde pública.
}

em tabela e figura e os achados qualitativos assumem a forma de categorização de falas. Essa análise quantiqualitativa se pautou pela literatura pertinente.

\section{RESULTADOS}

A população do estudo foi composta por 12 entrevistados (11 do sexo feminino e 1 do sexo masculino; 6 médicos e 6 enfermeiros); 2 participantes se encontravam na faixa etária de 20 a 29 anos; 7 na de 30 a 39 anos; 2 na de 40 a 49 anos; e 1 tinha 50 anos ou mais.

Em relação ao tempo de atuação em Viçosa do Ceará, todos os médicos relataram mais de 1 ano e menos de 5 anos. Vale ressaltar que 4 deles são profissionais estrangeiros inseridos no Programa Mais Médicos, do Governo Federal, instituído pela Lei n. 12.871/2013. Isso indica alta rotatividade de profissionais no município ${ }^{6}$.

Quanto aos enfermeiros, a atuação de 2 profissionais varia de 5 a 10 anos e a de 4 ultrapassa os 10 anos.

Todos os 12 participantes do estudo relataram prescrever fitoterápicos. Também informaram conhecer todos os medicamentos produzidos no município pelo Programa Farmácias Vivas.

Metade dos profissionais relatou prescrever fitoterápicos com muita frequência e a outra metade os prescreve com frequência regular.

Segundo 11 dos 12 participantes $(91,7 \%)$, a principal dificuldade para prescrever fitoterápicos se refere à indisponibilidade do medicamento em questão na unidade de farmácia viva e apenas 1 profissional $(8,3 \%)$ relatou ter conhecimento insuficiente no assunto, como se observa na Tabela 1. 
Tabela 1. Dados relativos à dificuldade na prescrição de fitoterápicos. Viçosa do Ceará, 2015.

\begin{tabular}{lcc}
\hline Dificuldades & Médicos & Enfermeiros \\
\hline Falta de fitoterápico & 5 & 6 \\
Conhecimento insuficiente sobre fitoterápicos & 1 & 0 \\
Não acredita na terapêutica & 0 & 0 \\
Descontinuidade no repasse do fitoterápico & 3 & 2 \\
\hline
\end{tabular}

Fonte: Elaborada pelos autores.

Os xaropes são os medicamentos mais prescritos, seguidos pelas cápsulas de maracujá e o elixir de aroeira. Já a tintura de malva santa e o sabonete de alecrim-pimenta são prescritos com menor frequência, como se observa na Figura 1.

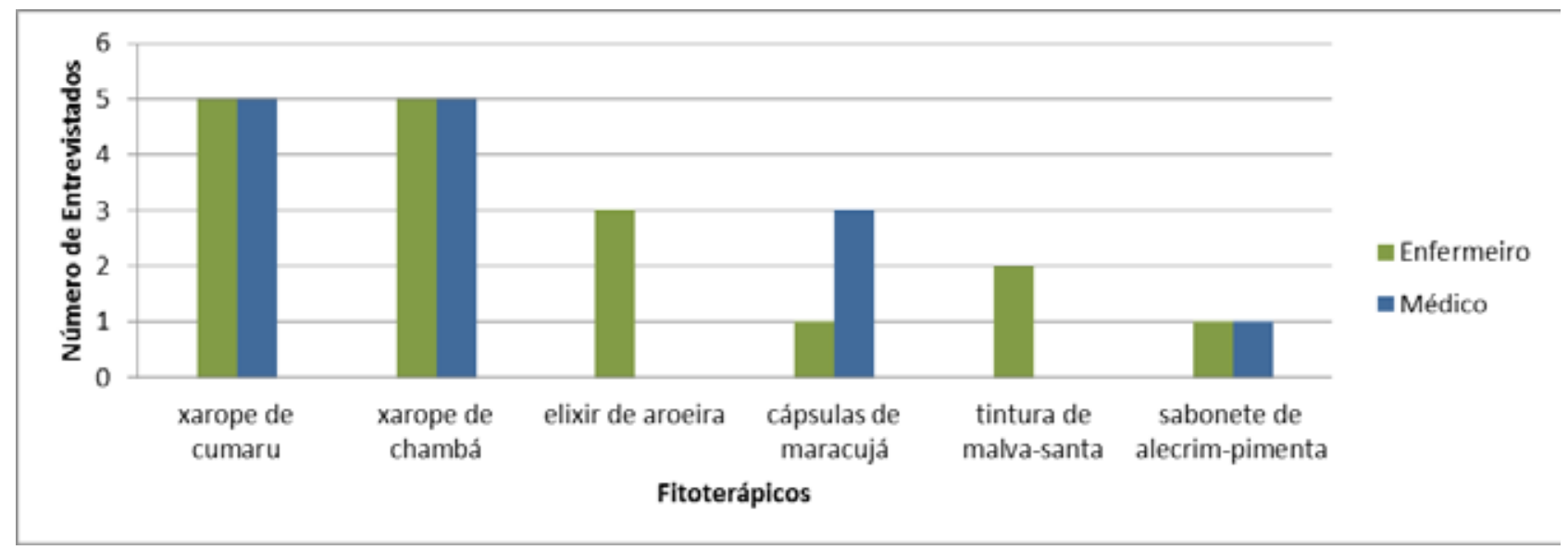

Figura 1. Distribuição dos fitoterápicos mais prescritos. Viçosa do Ceará, 2015.

Fonte: Elaborada pelos autores.

Os entrevistados relataram que a aceitação da prescrição de fitoterápicos por parte dos usuários do serviço de saúde é considerada boa, porém, 3 profissionais referiram certa resistência:

Uma parte dos pacientes aceita e outra parte não aceita. (Entrevistado 3)

Alguns apresentam certa resistência e outros, principalmente as mães, até preferem, pois acreditam não fazer mal aos filhos. (Entrevistado 10)

A maioria aceita, porém, às vezes, com pouca credibilidade. (Entrevistado 9)

Os demais profissionais relataram uma ótima aceitação, o que facilita a adesão ao tratamento e proporciona melhor resolutividade da patologia:

Os pacientes aceitam bem, muitas vezes pedem para prescrevermos fitoterápicos. (Entrevistado 8)

Quanto à eficácia dos fitoterápicos, todos os enfermeiros relataram ser ótima; entre os médicos, 4 indicaram ser ótima e 2 classificaram como boa.

Todos os profissionais informaram que a eficácia vem sendo avaliada por meio de acompanhamento clínico e relato do próprio usuário do serviço. Todos os médicos e 3 enfermeiros mostraram interesse em incluir outros fitoterápicos na farmácia viva, ao passo que 3 enfermeiros negaram interesse em disponibilizar novos fitoterápicos aos usuários. Para os profissionais da saúde, os fitoterápicos atuais são satisfatórios, porém, vários deles se encontram em falta, o que indica a necessidade de manter o estoque de fitoterápicos já usados no município.

É consensual entre os participantes o interesse em aperfeiçoar sua qualificação em fitoterápicos por meio de educação permanente. Eles destacam que receberam capacitação do farmacêutico responsável pelo centro fitoterápico, porém, tal treinamento não é permanente. Além disso, todos manifestam interesse em participar das atividades do programa de fitoterapia, principalmente de palestras educativas ( 5 médicos e 5 enfermeiros), trabalho com a comunidade ( 2 médicos e 2 enfermeiros) e 
acompanhamento clínico (2 médicos e 2 enfermeiros).

observou-se que metade dos profissionais prescreve fitoterápicos comercializados em farmácias comunitárias (2 enfermeiros e 4 médicos). A outra metade não prescreve (4 enfermeiros e 2 médicos), embora relatem conhecer outros fitoterápicos comercializados. $0 s$ principais medicamentos prescritos que não fazem parte do elenco de fitoterápicos da rede municipal são: Passiflora edulis Sims, Ginkgo biloba, Naturetti ${ }^{\circledR}$, Abrillar $^{\circledR}$, Hederax $^{\circledR}$ e Maracugina ${ }^{\circledR}$.

Quando indagados sobre os fitoterápicos que não prescrevem, 6 participantes relataram prescrever todos os medicamentos do elenco de fitoterápicos da farmácia básica, sendo que 2 não prescrevem tintura de alecrim-pimenta, 2 não prescrevem sachê para inalação, 1 não prescreve xarope de cumaru ou chambá e 1 não prescreve cápsulas de maracujá.

$0 s$ demais entrevistados citaram medicamentos que poderiam ser inseridos no elenco da farmácia básica do município, como xaropes sem adição de açúcar, para uso de diabéticos.

\section{DISCUSSÃO}

A composição das equipes de saúde é definida pela Portaria n. 2.488/2011 (normas e diretrizes para organização da atenção básica para a Estratégia Saúde da Família e para a Estratégia de Agentes Comunitários de Saúde) ${ }^{10}$.

Um estudo traçando o perfil dos trabalhadores da APS no Município de São Paulo detectou prevalência do sexo feminino e idades entre 21 e 40 anos $(50,5 \%)$ em uma amostra de 621 indivíduos. Cerca de $40 \%$ trabalhavam na UBS há mais de 5 anos e $37,9 \%$ trabalhavam na mesma UBS há menos de 5 anos ${ }^{11-13}$.

Categorizando o tempo no serviço por profissional, detectamos que os enfermeiros atuavam há mais de 5 anos (4 deles há mais de 10 anos) e 100\% dos médicos atuam há menos de 5 anos. Isso demonstra perfis profissionais diferentes: um contingente é antigo no serviço e o outro se encontra em renovação por meio do Programa Mais Médicos, do Governo Federal. Constatamos que muitos enfermeiros têm experiência com fitoterápicos desde o início da implantação do Programa Farmácias Vivas em Viçosa do Ceará, em $2002^{9}$.

Observa-se crescente aceitação da fitoterapia por parte dos profissionais da saúde, inclusive dos médicos ${ }^{14}$. Esse fato pode estar associado, entre outros aspectos, à comprovação científica

\section{... os fitoterápicos representam \\ $3 \%$ do mercado farmacêutico total no pais...}

das propriedades terapêticas de diversas plantas medicinais para o tratamento de determinadas patologias. De acordo com a literatura pertinente, esse é um fator que motiva as iniciativas para incluir a fitoterapia no âmbito do SUS ${ }^{4,15}$.

0 interesse no uso de plantas medicinais se relaciona ao alto custo dos medicamentos industrializados, à falta de acesso a assistência médica e farmacêutica, à crise econômica do país e à tendência dos consumidores optarem pelo uso de produtos naturais ${ }^{16-18}$.

os dados indicaram que os enfermeiros prescrevem mais fitoterápicos do que os médicos, corroborando os achados de um estudo realizado em Anápolis-G0. 0 posicionamento favorável ao uso de plantas medicinais e fitoterápicos diverge entre as categorias profissionais entrevistadas: $100 \%$ dos fisioterapeutas, farmacêuticos e odontólogos; $86 \%$ dos técnicos em enfermagem; $65 \%$ dos enfermeiros; e apenas $17 \%$ dos médicos ${ }^{19}$.

A Portaria MS n. 971/2006, que instituiu a PNPIC no SUS, trouxe novos subsídios para a normatização das ações governamentais na área de saúde, ampliando as opções terapêuticas com garantia de acesso às plantas medicinais e aos fitoterápicos ${ }^{20}$.

A falta de medicamentos e a descontinuidade em seu repasse para as farmácias são os grandes empecilhos para sua prescrição. A Sociedade Nacional de Agricultura indica que o Brasil, um dos países com maior biodiversidade do mundo, ainda encontra entraves para explorar todo o seu potencial no mercado de fitoterápicos ${ }^{21}$. Segundo estimativas da Associação Brasileira das Empresas do Setor Fitoterápico, Suplemento Alimentar e de Promoção da Saúde (Abifisa), os fitoterápicos representam $3 \%$ do mercado farmacêutico total no país, com faturamento de cerca de US\$ 1 bilhão e estimativa de crescimento anual em torno de 15\%. Em nível mundial, esse mercado movimenta anualmente cerca de US\$ 20 bilhões ${ }^{21}$. 
Alguns autores afirmam que há pouco apoio científico por parte de universidades; além disso, faltam iniciativas da gestão pública voltadas ao estudo e políticas públicas que garantam recursos para a implementação do uso de fitoterápicos e plantas medicinais no SUS 5 .

As classes profissionais prescritoras apresentaram pouca variedade entre os medicamentos mais frequentes. Tal fato também se deve à oferta nas UBS. A maior prescrição de xaropes expectorantes pode ser justificada pela prevalência de doenças do aparelho respiratório em determinadas faixas etárias. Atualmente, tais doenças constituem uma das principais causas de mortalidade e incapacidade no mundo, em especial quando afetam grupos populacionais vulneráveis (crianças e idosos). No Brasil, são a segunda causa mais comum de internação, superadas apenas pelo atendimento ao parto 22 .

0 desconhecimento dos efeitos indesejáveis de algumas plantas leva as pessoas a considerá-las inofensivas, recorrendo a elas por constituírem sabedoria popular, herança familiar, passada de geração a geração de povos e comunidades. Esse tipo de conhecimento é transmitido culturalmente e faz parte do senso comum, que busca opções naturais de baixo custo que não causem danos ao organismo ${ }^{23-24}$.

Concomitantemente ao uso de alopáticos, muitas vezes a população recorre a plantas medicinais desconhecendo: a existência de possivel interação, toxicidade e ação terapêutica comprovada; a forma correta de cultivo e preparo; e as indicações e contraindicações. Isso se deve ao fato das pessoas acreditarem que as plantas medicinais não podem vir a ser prejudiciais à saúde, independente da forma e da quantidade usada ${ }^{17}$.

A incorporação da fitoterapia nas UBS não representa somente a incorporação de plantas medicinais para a prevenção ou o alívio de enfermidades, algo que complemente ou substitua a alopatia, também envolve a valorização do conhecimento popular como um complexo de atitudes, valores e crenças que integram um estilo de vida. 0 trabalho com plantas medicinais cativa as pessoas, pois elas compartilham conhecimento - ao contrário do que ocorre com o tratamento à base de alopáticos ${ }^{4,25}$.

Segundo a Agência Nacional de Vigilância Sanitária (Anvisa), o fitoterápico é caracterizado pelo conhecimento da eficácia e dos riscos de seu uso, bem como pela reprodutibilidade e constância de sua

\section{... há 432 \\ fitoterápicos simples \\ e 80 compostos \\ registrados na \\ Anvisa.}

qualidade. Sua eficácia e segurança são validadas por meio de levantamentos etnofarmacológicos de uso, documentações tecnocientíficas ou evidências clínicas ${ }^{1}$.

Mesmo com a tecnologia avançada e com a medicina cada dia mais moderna, mostra-se necessário que os profissionais da saúde tenham capacitação quanto ao uso dos fitoterápicos, a fim de manter maior interação com a comunidade e aumentar sua afinidade com a cultura popular local, o que tende a melhorar adesão ao plano terapêutico fitoterápico ou alopático ${ }^{26}$.

A maioria dos programas de fitoterapia revisados por uma autora ${ }^{4}$ envolve um conjunto de medidas e ações de educação em saúde e de aproximação da comunidade - elas vão além da prescrição medicamentosa, identificando, apoiando e incentivando o uso e práticas caseiras e seguras para a população. Para exemplificar, alguns dos programas contemplam medidas que buscam trocar conhecimentos horizontais com a comunidade e orientá-la, quando necessário, em relação ao uso correto das plantas medicinais, por meio de palestras educativas, cartilhas, visitas domiciliares dos Agentes Comunitários de Saúde (ACS) e ações da equipe multidisciplinar das UBS.

A assistência a paciente, à família e à comunidade é uma função da equipe de saúde, que deve planejála com base na cultura da população e usar os recursos disponíveis para auxiliar a comunidade a melhorar seu nível de saúde. Para isso, deve-se ter conhecimentos sobre as propriedades terapêuticas das plantas medicinais, seu preparo, sua dosagem e sua indicação, além de compreender como as pessoas dessas comunidades percebem 0 processo saúdedoença ${ }^{27-28}$.

Em relação aos produtos industrializados, há 432 fitoterápicos simples e 80 compostos registrados na Anvisa. 0 setor produtivo industrial possui ampla legislação no tocante aos aspectos da produção e comercialização de fitoterápicos ${ }^{29}$.

As espécies de plantas medicinais com mais 
registros na Anvisa na forma de fitoterápicos são:

- Ginkgo biloba;

- Aesculus hippocastanum;

- Panax ginseng;

- Senna alexandrina;

- Peumus boldus Molina;

- Cynara scolymus;

- Passiflora edulis Sims;

- Valeriana officinalis L.; e

- Arnica montana L.

Essas espécies figuram entre as 34 previstas na lista de registro simplificado de fitoterápicos (Instrução Normativa n. 02/2014) - as quais têm registro facilitado por não precisarem comprovar critérios de segurança e eficácia terapêticas, por serem amplamente reconhecidas pela comunidade científica ${ }^{29}$.

0 desconhecimento e a falta de preparo profissional dificultam a adoção de plantas medicinais como recurso terapêutico na rede pública de saúde ${ }^{3}$. Isso abre amplo espaço para pesquisa, pois o uso de terapias menos dispendiosas para enfermidades crônico-degenerativas potencializa os investimentos humanos e financeiros destinados ao setor saúde. Assim, os benefícios dos fitoterápicos podem e devem ser combinados aos da terapia medicamentosa convencional, em linha com 0 princípio da integralidade da assistência à saúde no âmbito do SUS ${ }^{30}$.

\section{CONCLUSÃO}

0 uso de fitoterápicos é cada vez mais frequente no Brasil, principalmente na ESF, que adota essa prática de modo mais consistente no âmbito do SUS. No entanto, constatou-se a necessidade de novos estudos que analisem a aceitação do uso de fitoterápicos na APS, bem como sua prescrição por parte dos profissionais da saúde.

Outro ponto de vital importância deste estudo foi a prescrição per se: observou-se que a maioria dos profissionais da saúde já prescreve e relata dominar o uso de algum tipo de fitoterápico, o que mostra uma mudança em relação ao paradigma de uso da terapia alopática convencional.

Apesar do uso bem estabelecido, inclusive com livros já publicados na área de fitoterapia, e dos investimentos em unidades de farmácia viva, ainda se observa que os prescritores demandam formação
0 desconhecimento $e$ a falta de preparo profissional dificultam a adoção de plantas medicinais... específica para que a fitoterapia avance na APS. 0 s profissionais da saúde mostraram interesse em adquirir mais conhecimentos em fitoterapia para proporcionar melhor atendimento à população, complementando ou substituindo a terapia alopática convencional.

Conclui-se que o programa de fitoterapia de Viçosa do Ceará é bem aceito pelos profissionais das UBS. Assume destaque a disponibilidade dos fitoterápicos na unidade de farmácia viva, além da aceitação por parte dos usuários e do conhecimento dos prescritores sobre o assunto.

\section{CONTRIBUIÇÃO DOS AUTORES}

Ariel Átila Pontes Soares contribuiu com a realização da pesquisa, o delineamento do estudo e a redação do manuscrito. Ana Cecília Rodrigues e Silva contribuiu com o delineamento do estudo e a redação do manuscrito. Joaquim Horácio de Araújo Neto contribuiu com a redação do manuscrito. André Luiz Cunha Cavalcante e Olindina Ferreira Melo contribuíram com a redação e a revisão crítica do manuscrito. Rafaelly Maria Pinheiro Siqueira contribuiu com o delineamento do estudo e a revisão crítica do manuscrito.

\section{REFERÊNCIAS}

1. Brasil. Práticas integrativas e complementares: plantas medicinais e fitoterapia na atenção básica. Brasília (DF): Ministério da Saúde; 2012. (Cadernos de Atenção Básica, n. 31).

2. Organização Mundial da Saúde. A importância da farmacovigilância. Brasília (DF): 0MS; 2005.

3. Silvello CLC. 0 uso de plantas medicinais e de fitoterápicos no SUS: uma revisão bibliográfica [dissertation on the internet]. Porto Alegre: Universidade Federal do Rio Grande do Sul; 2010 [cited 2018 May 8]. Available from: http://www.lume.ufrgs. br/bitstream/handle/10183/28232/000769371. pdf? sequence $=1$ 
4. Becker MM. Programas de fitoterapia na rede pública de saúde no Brasil [monograph on the internet]. Florianópolis: Universidade Federal de Santa Catarina; 2012 [cited 2018 May 8]. Available from:

Mariana-Becker.pdf

5. Matos FJA. Plantas medicinais: guia de seleção e emprego de plantas usadas em fitoterapia no Nordeste do Brasil. 3. ed. Fortaleza: Ed. UFC; 2007.

6. Brasil. Lei n. 12.871, de 22 de outubro de 2013. Institui o Programa Mais Médicos, altera as Leis $n$. 8.745, de 9 de dezembro de 1993, e n. 6.932, de 7 de julho de 1981, e dá outras providências. Diário Oficial da União, Brasília (2013 0ct 23); Sec 1.

7. Alexandre RF, Garcia FN, Simões CMO. Fitoterapia baseada em evidências. Parte 1: medicamentos fitoterápicos elaborados com ginko, hipérico, kava e valeriana. Acta Farm Bonaer [serial on the internet]. 2005 [cited 2018 May 8];24(2):300-9. Available from: http://www.latamjpharm.org/trabajos/24/2/ LAJOP $24 \begin{array}{lllllll}24 & 7 & 1 & \text { YSOCRS9CK3.pdf }\end{array}$

8. Nascimento WMC, Melo OF, Silva IF, Souza FL. Plantas medicinais e sua utilização pelas comunidades do município de Sobral, Ceará. Sanare (Sobral, Online) [serial on the internet]. 2013 [cited 2018 0ct 25];12(1):46-53. Available from: https://sanare. emnuvens.com.br/sanare/article/view/328/262

9. Fonseca SGC, Moura LC, organizer. Memento fitoterápico. Viçosa do Ceará (CE): Prefeitura de Viçosa do Ceará; 2002.

10. Brasil. Portaria n. 2.488 , de 21 de outubro de 2011. Aprova a Política Nacional de Atenção Básica, estabelecendo a revisão de diretrizes e normas para a organização da Atenção Básica, para a Estratégia Saúde da Família - ESF e o Programa de Agentes Comunitários de Saúde - PACS. Diário Oficial da União, Brasília (2011 0ct 24); Sec 1.

11. España. Base de Datos de Profesionales Sanitarios Colegiados. Madrid: Instituto Nacional de Estadística; 2011.

12. Carrillo-García C, Solano-Ruíz MC, MartínezRoche ME, Gómez-García CI. Influência do gênero e da idade: satisfação no trabalho de profissionais da saúde. Rev Latinoam Enferm [serial on the internet]. 2013 [cited 2018 May 8];21(6):1314-20. Available from: $\quad$ http://www.scielo.br/pdf/rlae/v21n6/ pt 0104-1169-rlae-21-06-01314.pdf

13. Marsiglia RMG. Perfil dos trabalhadores da atenção básica em saúde no município de São Paulo: região norte e central da cidade. Saúde Soc [serial on the internet]. 2011 [cited 2018 May 8];20(4):900-11. Available from: http://www.scielo.br/pdf/sausoc/ v20n4/08.pdf
14. Akiyama K. Práticas não convencionais em medicina no Município de São Paulo [thesis on the internet]. São Paulo: Universidade de São Paulo; 2004 [cited 2018 Nov 16]. Available from: file:///D:/ KazuseiAkiyamaTeseD02004.pdf

15. Fachina F, Abelan US. Uso e aceitação de medicamentos magistrais em pacientes atendidos nas Clínicas Integradas - Unirp de São José do Rio Preto, SP. Rev Bras Farm [serial on the internet]. 2012 [cited 2018 May 8];93(2):167-72. Available from: http://www.rbfarma.org.br/files/rbf-2012-932-6.pdf

16. Simões CM0, Schenkel EP, Gosmann G, Mello JCP, Mentz LA, Petrovick PR. Farmacognosia: da planta ao medicamento. Florianópolis: Ed. UFSC; 1999.

17. Barreto BB. Fitoterapia na atenção primária à saúde: a visão dos profissionais envolvidos [dissertation on the internet]. Juiz de Fora (MG): Universidade Federal de Juiz de Fora; 2011 [cited 2018 Nov 16]. Available from: http:// www.ufjf.br/pgsaudecoletiva/files/2011/03/ DISSERTA\%C $3 \% 87 \%$ C 3\%830-BENILSON-versao-final. $\mathrm{pdf}$

18. Gerhardt TE. Itinerários terapêuticos em situações de pobreza: diversidade e pluralidade. Cad Saúde Pública [serial on the internet]. 2006 [cited 2018 May 8];22(11):2449-63. Available from: http:// www.scielo.br/pdf/csp/v22n11/19.pdf

19. Dutra MG. Plantas medicinais, fitoterápicos e saúde pública: um diagnóstico situacional em Anápolis, Goiás [dissertation on the internet]. Juiz de Fora (MG): Universidade Federal de Juiz de Fora; 2009 [cited 2018 May 8]. Available from: http://www.ufjf.br/pgsaudecoletiva/files/2011/03/ DISSERTA \%C $3 \% 87 \%$ C $3 \% 830$-BENILSON-versao-final. $p d f$

20. Brasil. Política Nacional de Práticas Integrativas e Complementares: atitude de ampliação de acesso [document on the internet]. Brasília (DF): Ministério da Saúde; 2006 [cited 2018 May 8]. Available from: http://bvsms.saude.gov.br/bvs/publicacoes/pnpic. pdf

21. Sociedade Nacional de Agricultura. Setor de fitoterápicos sofre com a falta de mão de obra especializada e investimento [document on the internet]. 2014 [cited 2018 May 8]. Available from: http://www.sna.agr.br/setor-de-fitoterapicosenfrenta-falta-de-mao-de-obra-especializada-einvestimento/

22. Cajazeira AA. Geografia, ambiente e saúde: correlações entre o clima e a incidência de doenças respiratórias em Maracanaú/CE [dissertation on the internet]. Fortaleza: Universidade Federal do Ceará; 2013 [cited 2018 May 8]. Available from: http://www. repositorio.ufc.br/bitstream/riufc/7357/1/2012 dis aacajazeira.pdf 
23. Faria PG, Ayres A, Alvim NAT. 0 diálogo com gestantes sobre plantas medicinais: contribuições para os cuidados básicos de saúde. Acta Sci, Health Sci [serial on the internet]. 2004 [cited 2018 May 8];26(2):287-94. Available from: http://periodicos. uem.br/ojs/index.php/ActaSciHealthSci/article/ view/1579/931

24. Machado DC, Czermainski Silvia BC, Lopes EC. Percepções de coordenadores de unidades de saúde sobre a fitoterapia e outras práticas integrativas e complementares. Saúde Debate [serial on the internet]. 2012 [cited 2018 May 8];36(95):615-23. Available from: http://www.scielo.br/pdf/sdeb/ v36n95/a13v36n95.pdf

25. Diniz MFFM, Oliveira RAG, Medeiros ACD, Malta Júnior A. Memento fitoterápico: as plantas como alternativa terapêutica, conhecimentos populares e científicos. João Pessoa: Universitária; 1997.

26. Tôrres AR, Oliveira RAG, Diniz MFFM, Araújo EC. Estudo sobre o uso de plantas medicinais em crianças hospitalizadas da cidade de João Pessoa: riscos e benefícios. Rev Bras Farmacogn [serial on the internet]. 2005 [cited 2018 May 8];15(4):37380. Available from: http://www.scielo.br/pdf/rbfar/ v15n4/a18v15n4.pdf

27. Silva MIG, Gondim APS, Nunes IFS, Sousa FCF. Utilização de fitoterápicos nas unidades básicas de atenção à saúde da família no Município de Maracanaú (CE). Rev Bras Farmacogn [serial on the internet]. 2006 [cited 2018 May 8];16(4):455-62. Available from: http://www.scielo.br/pdf/rbfar/ v16n4/a03v16n4.pdf

28. Santos RL, Guimaraes GP, Nobre MSC, Portela AS. Análise sobre a fitoterapia como prática integrativa no Sistema Único de Saúde. Rev Bras Plantas Med [serial on the internet]. 2011 [cited 2018 May 8];13(4):486-91. Available from: http://www.scielo. br/pdf/rbpm/v13n4/a14v13n4.pdf

29. Brasil. Instrução Normativa n. 2, de 13 de maio de 2014. Publica a "Lista de medicamentos fitoterápicos de registro simplificado" e a "Lista de produtos tradicionais fitoterápicos de registro simplificado". Diário Oficial da União, Brasília, (2014 May 14); Sec 1.

30. Borges KB, Bautista BH, Guilera S. Diabetes: utilização de plantas medicinais como forma opcional de tratamento. Rev Eletrônica Farm [serial on the internet]. 2008 [cited 2018 May 8];5(2):1220. Available from: https://revistas.ufg.br/REF/ article/view/5149/4256
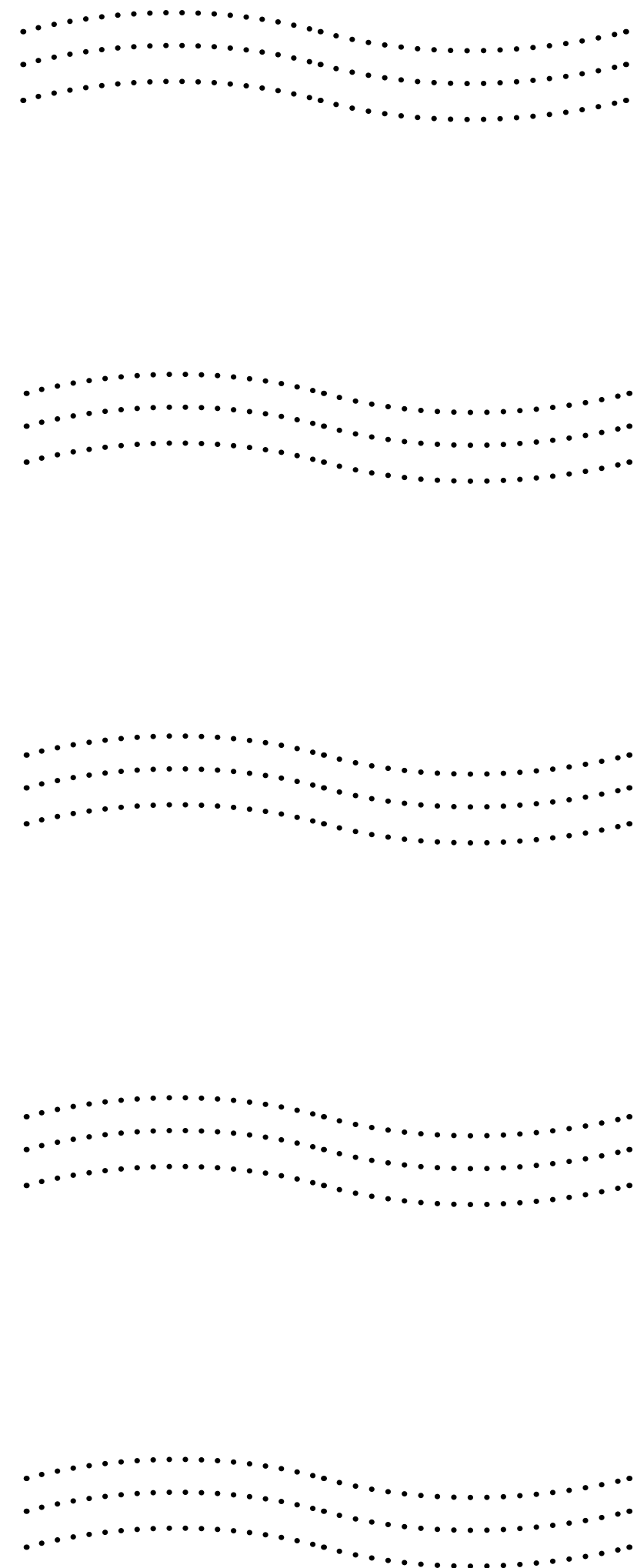